\title{
Pourquoi devrait-on inscrire les études cliniques?
}

$\mathrm{U}$ $\mathrm{n}$ de nos amis envisage de participer à une étude multicentrique de phase II contrôlée par placebo et portant sur un médicament contre le cancer du poumon à petites cellules. Même si elles sont détaillées et claires, les feuillets d'information des patients ne contiennent aucune promesse au sujet de la publication des résultats de l'étude ou de leur mise à la disposition du public, voire même des chercheurs locaux ayant participé à l'étude. Il est peu probable toutefois que notre ami, face à son état et à son sombre pronostic, pose des questions à ce sujet. Il sait qu'il a probablement peu de chance d'en tirer un avantage important pour lui-même (il peut se retrouver dans le groupe placebo), que les risques sont importants (s'il reçoit en fait le médicament), sans oublier les inconvénients et la fatigue inévitables causés par les visites à la clinique et les examens plus nombreux. Comme beaucoup de patients qui participent à des études cliniques, il est toutefois motivé par l'espoir altruiste de contribuer à un effort qui pourrait un jour aider des patients comme lui. Comme la plupart des gens, il suppose que quels que soient les résultats de l'étude, il y aura des retombées sur les connaissances scientifiques.

Ce dont notre ami ne tient peut-être pas compte, c'est que la croissance des connaissances est entravée par le fait que beaucoup de données issues d'études cliniques ne sont jamais dévoilées. Il se peut que les résultats de son étude ne soient pas publiés, pour diverses raisons, comme un nombre insuffisant de patients recrutés, des coupures de financement, des découvertes parallèles rendant l'étude non pertinente ou, ce qui est le plus probable, parce que les résultats de l'étude se révèlent défavorables pour le produit du commanditaire. Or, si l'étude demeure non publiée, le public, les médecins, les rédacteurs, les critiques systématiques et les méta-analystes ne sauront même pas qu'elle a eu lieu — sauf si on l'a inscrite à un registre accessible des études.

Dans ce numéro (voir page 1700)1 ${ }^{1}$ le Comité international des rédacteurs de revues médicales (CIRRM) réitère et clarifie sa déclaration précédente ${ }^{2}$ selon laquelle l'inscription des études cliniques dès leur début deviendra, à compter du $1^{\mathrm{er}}$ juillet 2005, une condition de publication. La non-inscription d'une nouvelle étude à compter de cette date obligera le $7 A M C$ et d'autres journaux membres du CIRRM, ainsi que des dizaines d'autres journaux qui appuient la déclaration originale sur l'inscription, à refuser d'envisager la publication du document qui en découlera.

L'inscription des études offre de nombreux avantages. Elle aide des patients à trouver des études auxquelles ils pourraient être admissibles et qui pourraient leur donner accès à de nouvelles thérapies. Comme les études sont inscrites, il est plus difficile de dissimuler l'existence d'études en cours, ce qui aide à contrer les profils déformés d'efficacité et d'innocuité découlant de la publication sélective de résultats d'études. Le registre aide aussi à évaluer des études terminées. Examinateurs pairs, rédacteurs et commentateurs, ainsi que médecins et patients, peuvent ainsi non seulement étudier les travaux publiés dans un domaine donné, mais aussi voir ce qui s'an- nonce sur le plan des études. Des rédacteurs peuvent, par exemple, étudier un rapport d'étude sur un médicament qui montre un avantage clinique par rapport au placebo, mais aussi un risque important mais non significatif d'événements indésirables graves. Une recherche effectuée dans le registre public des études pourrait révéler l'existence d'une étude semblable comportant un échantillon d'une taille suffisante pour produire des estimations plus précises de la fréquence des événements indésirables. On pourrait recommander aux cliniciens et aux patients qui sont au courant de l'étude en cours d'en attendre les résultats avant d'utiliser le nouveau médicament. Les multiples études en cours sur les inhibiteurs de la COX-2 sont de bons exemples récents.

De même, les critiques systématiques et les méta-analystes qui connaissent l'existence d'études en cours et la taille de leur échantillon peuvent poser des hypothèses au sujet des effets que les données à venir pourraient avoir sur leurs conclusions et leurs analyses. Les organismes subventionnaires du secteur public et les sociétés pharmaceutiques qui commanditent des études peuvent consulter un registre d'études en cours lorsqu'ils doivent prendre des décisions sur le financement de nouvelles études.

Les sociétés pharmaceutiques qui subventionnent la majorité des études cliniques ont soutenu que la divulgation de leurs études en cours dans un registre révélerait des objectifs commerciaux secrets, empiéterait sur les droits de propriété intellectuelle, nuirait à la valeur de leurs actions et refroidirait l'intérêt des investisseurs. D'autres soutiennent toutefois qu'à cause de leur nature même, les études cliniques (multicentriques, mettant à contribution de multiples chercheurs et des centaines de patients, et scrutées continuellement par les analystes de Wall Street) sont déjà «publiques» et que l'argument commercial en faveur de la protection de leur secret est en grande partie spécieux. De plus, lorsque tous les intervenants se conforment aux mêmes règles de divulgation, aucun d'entre eux n'est désavantagé.

La situation actuelle, dans le contexte de laquelle les études cliniques portant sur des sujets humains sont dissimulées par le voile de «l'intérêt exclusif» et sont assujetties à l'examen scientifique seulement si leurs commanditaires commerciaux le veulent bien, est inacceptable pour des raisons éthiques et scientifiques. Il doit exister un meilleur équilibre entre la volonté de garantir la viabilité commerciale des activités de $\ll \mathrm{R}-\mathrm{D}$ » et celle de protéger les meilleurs intérêts des patients et du public. L'inscription publique des études cliniques est un moyen de rétablir l'équilibre. $-7 A M C$

\section{Références}

1. De Angelis CD, Drazen JM, Frizelle FA, Haug C, Hoey J, Horton R, et al. Is this clinical trial fully registered? A statement from the International Committee of Medical Journal Editors [editorial]. FAMC 2005;172(13):1700-2.

2. De Angelis C, Drazen JM, Frizelle FA, Haug C, Hoey J, Horton R, et al. Clinical trial registration: a statement from the International Committee of Medical Journal Editors [editorial]. FAMC 2004;171(6):606-7. 